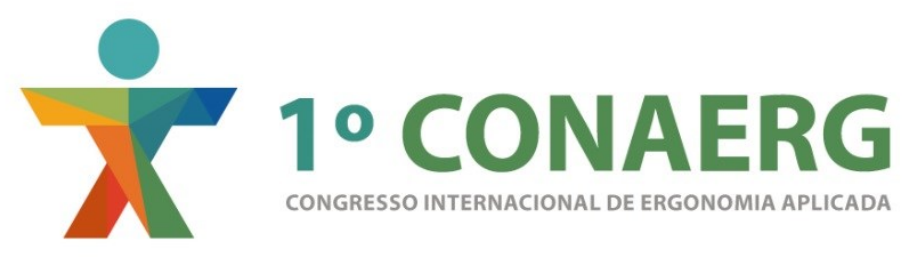

\title{
CONDIÇÕES ERGONÔMICAS DOS POSTOS DE TRABALHO NO CENTRO DE PROCESSAMENTO DE DADOS DO TRIBUNAL DE JUSTIÇA DE SERGIPE
}

\author{
Maria Goretti Fernandes (1) \\ Anne Kelly do Carmo Santana (2) \\ Elizabeth Christina Viana Augusto (3) \\ Geraldo Magella Teixeira (4)
}

(1) Universidade Federal de Sergipe - UFS, Professora Efetiva da UFS, Doutora em

Ciências da Saúde pela Universidade Federal do Rio Grande do Norte

Email: fisio100@yahoo.com.br

(2) Universidade Federal de Sergipe, Graduada em Fisioterapia

Email: acksantana@hotmail.com

(3) União Metropolitana de Educação e Cultura, Especialista em Traumato-ortopedia

Email: bethviana@oi.com.br

(4) Universidade Estadual de Ciências da Saúde de Alagoas - UNCISAL, Professor Efetivo da UNCISAL, Doutor pela Universidade Federal de São Paulo

Email: magellafisio@hotmail.com

\begin{abstract}
RESUMO
O propósito do estudo é verificar os sintomas osteomusculares dos trabalhadores do Tribunal de Justiça de Sergipe, bem como as condições ergonômicas nos postos de trabalho e observar se há correlação entre essas variáveis. Foi utilizada a avaliação das condições ergonômicas em postos de trabalho e ambientes informatizados para coletar os dados dos locais de trabalho. A prevalência de sintomas osteomusculares foi maior nas regiões lombar e torácica. Sugere-se que o mobiliário inadequado dos postos de trabalho, o sobrepeso dos trabalhadores e o tempo em que o funcionário exerce a mesma função estejam relacionados com os distúrbios osteomusculares.
\end{abstract}

\section{ABSTRACT}

The purpose of the study is to verify the musculoskeletal symptoms of workers Court of Sergipe and ergonomic conditions in the workplace and see if there is a correlation between these variables. A plug for characterization of the sample was used, the Nordic Musculoskeletal Questionnaire as an identification tool for musculoskeletal symptoms and Checklist for assessment of ergonomic conditions in jobs and computing environments to collect data from workplaces. The prevalence of musculoskeletal symptoms was higher in the lumbar, thoracic regions. It is suggested that the furniture, overweight and time in which the official serves the same function are related to musculoskeletal disorders.

\section{INTRODUÇÃO}


Saúde do trabalhador, conforme descreve o Conselho Nacional de Saúde, pode ser entendida como um conjunto de atividades que se destina, através das ações de vigilância epidemiológica e vigilância sanitária, à promoção e proteção da saúde dos trabalhadores, assim como visa à recuperação e reabilitação da saúde dos trabalhadores submetidos aos riscos e agravos advindos das condições de trabalho (CONSELHO NACIONAL DE SAÚDE, 1990).

Mendes (1991), em seu trabalho de revisão, apresentou a linha do tempo do binômio saúdetrabalho expondo a evolução do campo de estudo, inicialmente como Medicina do Trabalho, denominada posteriormente de Saúde Ocupacional e, atualmente, conhecida como Saúde do trabalhador. Para o autor, esta última surgiu de insuficiências do campo da Saúde Ocupacional, dentre as quais se encontravam a centralização da figura do médico e a sua manutenção no âmbito do trabalho em detrimento do setor de saúde.

Estudos têm apontado a associação entre sintomas osteomusculares, dor, desconforto e parestesia que são reportados pelos trabalhadores e suas atividades laborais. Para as investigações a cerca desses distúrbios o Questionário Nórdico de Sintomas Osteomusculares (QNSO) é reconhecido internacionalmente como o padrão ouro, e têm sido frequentemente utilizado na maior parte dos estudos relacionados à área (MARTARELLO, 2009; VITTA et al., 2012; LEMOS, 2014).

Fortemente ligadas à Saúde do Trabalhador encontram-se as questões ergonômicas oferecidas pelo ambiente laboral. Na conceituação da International Ergonomics Association (IEA), ergonomia é a disciplina científica relacionada com a compreensão das interações entre o homem e outros elementos do Sistema, tendo como objetivo proporcionar o bem estar do indivíduo e assim trazer melhorias para sua performance. (IEA, 2015). Essa definição também é adotada pela Associação Brasileira de Ergonomia (ABERGO) e pela Human Factors and Ergonomis Society (ABERGO, 2015; HFES, 2015; MÁSCULO e VIDAL, 2011, p.113).

Investigações sobre as condições ergonômicas em postos de trabalho nos quais se utiliza computador têm sugerido que somente a adequação do mobiliário, de acordo com as normas regulamentadoras, não é suficiente para evitar as disfunções osteomusculares nos trabalhadores (ROBOREDO e POLISSENI, 2006; ROSA et al., 2009).

A produção científica em saúde do trabalhador tem crescido no Brasil. Entre 2001 e 2008 foram desenvolvidos, principalmente, os estudos com método de abordagem quantitativo cujos objetos de estudo são discussões conceituais das relações saúde-ambiente-trabalho. Os profissionais da saúde compõem a população mais estudada, enquanto os artigos envolvendo os trabalhadores do setor de serviço possuem menor número de publicações (BEZERRA, 2010).

O campo Saúde do Trabalhador, podendo-se incluir a ergonomia, é uma área de pesquisa em ascensão e estudos que relacionem as atividades laborais e as condições oferecidas pelo posto de trabalho podem embasar a necessidade das intervenções cinesioterapêuticas e ergonômicas nas empresas.

Diante deste contexto, o referido estudo se propõe a verificar os sintomas osteomusculares dos servidores do centro de processamento de dados do Tribunal de Justiça de Sergipe (TJSE), bem como as condições ergonômicas nos postos de trabalho.

\section{METODOLOGIA}

O trabalho trata-se de estudo observacional analítico, do tipo transversal, a ser realizado na instituição pública, TJSE, da cidade de Aracaju no estado de Sergipe. A amostra foi constituída por conveniência, composta por trabalhadores do setor administrativo, acima de 18 anos de idade, de ambos os sexos, com tempo mínimo na mesma função de um ano e que fazem uso de computador em sua rotina de atividades laborais. Foram excluídos os 
trabalhadores com diagnóstico de lesão por esforço repetitivo (LER) e doenças osteomusculares relacionadas ao trabalho (DORT).

Foi elaborada uma ficha de caracterização da amostra composta de duas seções. A primeira incluiu os dados antropométricos: idade, sexo, altura e índice de massa corporal (IMC). A segunda, os dados sócio-ocupacionais: prática de atividade física regular, tempo de em que desempenha a mesma função, carga horária de trabalho, ocorrência de algum acidente com comprometimento osteomuscular recente.

A identificação dos sintomas musculoesqueléticos foi realizada através da aplicação do Questionário Nórdico de Sintomas Osteomusculares (QNSO), elaborado por Kuorinka et al. (1987), com versão brasileira validada por Pinheiro et al. (2002). O QNSO contém uma representação da região posterior humana, dividida em nove pontos: região da coluna cervical, ombros, coluna dorsal, cotovelos, punhos e mãos, coluna lombar, quadril e coxa, joelhos, tornozelos e pés. Essa verificação foi feita por meio de entrevista com os trabalhadores, visando minimizar erros de resposta.

Quanto à avaliação dos postos de trabalho, esta se realizou por meio da versão de 2014 Cheklist para avaliação das condições ergonômicas em postos de trabalho e ambientes informatizados, desenvolvido por Couto e colaboradores. Essa ferramenta é composta por 13 itens, os quais contemplam perguntas sobre o ambiente de trabalho, cujas respostas são dadas como Não (0) E Sim/Não se aplica (1). O escore pode ser gerado a partir da soma dos pontos de cada item isoladamente ou na totalidade do cheklist, por meio do seguinte escalonamento: $100 \%$ a $91 \%$ é classificado como condição ergonômica excelente; $90 \%$ a $71 \%$ é uma boa condição ergonômica; $70 \%$ a $51 \%$ a condição ergonômica é razoável; $50 \%$ a $31 \%$ demonstra condição ergonômica ruim e menos que $31 \%$ a condição ergonômica é péssima.

As informações coletadas foram tabuladas no programa Microsoft Excel ${ }^{\circledR} 2010$. Os dados foram descritos por meio de frequência simples e percentual quando categóricas; média e desvio padrão quando contínuas ou discretas. Para avaliar associação entre variáveis categóricas foi utilizado o teste qui-quadrado de Pearson com correção de continuidade. Para avaliar diferenças de média foi utilizado o teste de Mann-Whitney e tamanhos de efeitos foram calculados quando havia diferença significativa. O nível de significância adotado foi de $5 \%$ e o software utilizado foi o R Core Team ${ }^{\circledR} 2016$. O tamanho de efeito para testes Qui-Quadrado (W) foi classificado como: $\leq 0,10$ : capaz de detectar pequenas diferenças; 0,1 a 0,3: capaz de detectar diferenças medianas;0,3 a 0,5: capaz de detectar grandes diferenças.

\section{RESULTADOS}

Quanto aos resultados encontrados foram entrevistados 69 funcionários. Destes, 5 foram excluídos do estudo, pois desempenhavam a função há menos de 1 ano e 1 devido à gestação. A caracterização da amostra é apresentada na Tabela 1.

Tabela 1. Caracterização da amostra dos Trabalhadores do TJSE.

\begin{tabular}{ccc}
\hline & $\mathrm{N}$ & $\%$ \\
\hline Gênero & & \\
Feminino & 23 & 37 \\
Masculino & 40 & 63 \\
$\mathrm{CH}$ & 31 & 3 \\
& Média & DP
\end{tabular}




\begin{tabular}{ccc} 
Idade & 33,63 & 8,23 \\
Peso & 75,48 & 16,88 \\
Altura & 1,71 & 0,13 \\
IMC & 25,85 & 5,34 \\
At Física & 34,00 & 53,97 \\
$\begin{array}{c}\text { Tempo na função } \\
\text { anos }\end{array}$ & 8,84 & 6,63 \\
\hline
\end{tabular}

A tabela 2 demonstra a frequência simples encontrada na classificação ergonômica dos postos de trabalho em cada item do Checklist com relação aos itens avaliados pelos pesquisadores.

Tabela 2. Frequência da classificação ergonômica quanto aos itens avaliados

\begin{tabular}{cccccc}
\hline & Excelente & Boa & Razoável & Ruim & Péssima \\
\hline Cadeira & 63 & 0 & 0 & 0 & 0 \\
Mesa de Trabalho & 0 & 0 & 63 & 0 & 0 \\
Apoio para os Pés & 0 & 63 & 0 & 0 & 0 \\
Teclado & 61 & 2 & 0 & 0 & 0 \\
Monitor & 0 & 21 & 42 & 0 & 0 \\
Gabinete e CPU & 37 & 0 & 26 & 0 & 0 \\
Sistema de Trabalho & 63 & 0 & 0 & 0 & 0 \\
Acessibilidade & 63 & 0 & 0 & 0 & 0
\end{tabular}

Quanto aos sintomas relatados pelos trabalhadores, a tabela 3 apresenta a frequência simples e a porcentagem do Questionário Nórdico de Sintomas Osteomusculares.

Tabela 3. Resultado do QNSO

\begin{tabular}{cccc}
\hline & 1 ano & 7 dias & Afastamento \\
& $\mathrm{N}(\%)$ & $\mathrm{N}(\%)$ & $\mathrm{N}(\%)$ \\
\hline Pescoço & $37(59)$ & $21(33)$ & $4(6)$ \\
Ombro & $23(37)$ & $14(22)$ & $4(6)$ \\
Cotovelo & $6(10)$ & $5(8)$ & $3(5)$ \\
Antebraço & $11(17)$ & $9(14)$ & $3(5)$ \\
Punhos mãos & $26(41)$ & $15(24)$ & $7(11)$ \\
dedos & & & \\
Região dorsal & $29(46)$ & $23(37)$ & $7(11)$ \\
Região lombar & $31(49)$ & $24(38)$ & $5(8)$ \\
Quadris coxas & $9(14)$ & $5(8)$ & $1(2)$ \\
Joelhos & $15(24)$ & $10(16)$ & $1(2)$ \\
Tornozelos pés & $6(10)$ & $5(8)$ & $2(3)$ \\
\hline
\end{tabular}


As tabelas 4 e 5 representam o resultado dos testes de correlação entre as características da amostra e os sintomas osteomusculares do QNSO, em que ocorreu diferença estatisticamente significativa em pelo menos um item.

Tabela 4. Correlação entre os dados antropométricos dos trabalhadores e o QNSO

\begin{tabular}{|c|c|c|c|}
\hline & $\begin{array}{c}1 \text { ano } \\
\text { Média (DP) }\end{array}$ & $\begin{array}{c}7 \text { dias } \\
\text { Média (DP) }\end{array}$ & $\begin{array}{l}\text { Afastamento } \\
\text { Média (DP) }\end{array}$ \\
\hline \multicolumn{4}{|l|}{ Pescoço } \\
\hline \multicolumn{4}{|l|}{ Idade } \\
\hline Sem Dor & $33,42(8,08)$ & $33,71(7,93)$ & $33,66(8,4)$ \\
\hline Com Dor & $33,78(8,45)$ & $33,48(9,01)$ & $33,25(6,13)$ \\
\hline$p$-valor & 0,911 & 0,640 & 0,817 \\
\hline W & - & - & - \\
\hline \multicolumn{4}{|l|}{ Peso } \\
\hline Sem Dor & $78,42(19,89)$ & $77,33(17,76)$ & $74,41(16,71)$ \\
\hline Com Dor & $73,41(14,32)$ & $71,76(14,65)$ & $91,25(11,64)$ \\
\hline p-valor & 0,476 & 0,223 & 0,041 \\
\hline W & - & - & 0,256 \\
\hline \multicolumn{4}{|l|}{ Altura } \\
\hline Sem Dor & $1,7(0,16)$ & $1,71(0,14)$ & $1,7(0,13)$ \\
\hline Com Dor & $1,71(0,11)$ & $1,7(0,1)$ & $1,81(0,04)$ \\
\hline p-valor & 0,894 & 0,369 & 0,068 \\
\hline W & - & - & - \\
\hline \multicolumn{4}{|l|}{ IMC } \\
\hline Sem Dor & $27,26(7,33)$ & $26,4(6,12)$ & $25,71(5,47)$ \\
\hline Com Dor & $24,87(3,07)$ & $24,75(3,14)$ & $27,94(2,55)$ \\
\hline $\mathrm{p}$-valor & 0,738 & 0,710 & 0,083 \\
\hline W & - & - & - \\
\hline \multicolumn{4}{|l|}{$\mathrm{CH}$} \\
\hline Sem Dor & $31,15(3,26)$ & $30,95(3,7)$ & $30,85(3,37)$ \\
\hline Com Dor & $30,54(3,29)$ & $30,48(2,18)$ & $30(0)$ \\
\hline$p$-valor & 0,473 & 0,565 & 0,796 \\
\hline W & - & - & - \\
\hline \multicolumn{4}{|c|}{ Tempo na função anos } \\
\hline Sem Dor & $10,1(7,5)$ & $9,86(7,03)$ & $9,03(6,78)$ \\
\hline Com Dor & $7,96(5,89)$ & $6,81(5,32)$ & $6(2,71)$ \\
\hline p-valor & 0,293 & 0,112 & 0,596 \\
\hline W & - & - & - \\
\hline
\end{tabular}

Teste de Mann-Whitney; W - Tamanho de efeito; $\mathrm{CH}$ - Carga Horária 
Tabela 5. Correlação entre os dados antropométricos dos Trabalhadores e o QNSO

\begin{tabular}{|c|c|c|c|}
\hline & $\begin{array}{c}1 \text { ano } \\
\text { Média (DP) }\end{array}$ & $\begin{array}{c}7 \text { dias } \\
\text { Média (DP) }\end{array}$ & $\begin{array}{l}\text { Afastamento } \\
\text { Média (DP) }\end{array}$ \\
\hline \multicolumn{4}{|l|}{ Ombro } \\
\hline \multicolumn{4}{|l|}{ Idade } \\
\hline Sem Dor & $32,03(7,92)$ & $32,33(7,45)$ & $33,56(8,26)$ \\
\hline Com Dor & $36,43(8,18)$ & $38,21(9,44)$ & $34,75(9,03)$ \\
\hline p-valor & 0,028 & 0,047 & 0,734 \\
\hline W & 0,277 & 0,250 & - \\
\hline \multicolumn{4}{|l|}{ Peso } \\
\hline Sem Dor & $75,05(15,2)$ & $77,78(17,4)$ & $75,68(17,11)$ \\
\hline Com Dor & $76,22(19,81)$ & $67,43(12,3)$ & $72,5(14,64)$ \\
\hline p-valor & 0,808 & 0,044 & 0,734 \\
\hline \multirow[t]{2}{*}{ W } & & & - \\
\hline & - & 0,273 & \\
\hline \multicolumn{4}{|l|}{ Altura } \\
\hline Sem Dor & $1,69(0,14)$ & $1,71(0,14)$ & $1,71(0,13)$ \\
\hline Com Dor & $1,74(0,11)$ & $1,7(0,09)$ & $1,75(0,15)$ \\
\hline p-valor & 0,230 & 0,487 & 0,470 \\
\hline W & - & - & - \\
\hline \multicolumn{4}{|l|}{ IMC } \\
\hline Sem Dor & $26,34(5,71)$ & $26,57(5,64)$ & $26,01(5,47)$ \\
\hline Com Dor & $25,01(4,64)$ & $23,34(3,14)$ & $23,56(2,19)$ \\
\hline p-valor & 0,420 & 0,062 & 0,374 \\
\hline W & - & - & - \\
\hline \multicolumn{4}{|l|}{$\mathrm{CH}$} \\
\hline Sem Dor & $30,5(3,16)$ & $31,02(3,68)$ & $30,68(3,14)$ \\
\hline Com Dor & $31,3(3,44)$ & $30(0)$ & $32,5(5)$ \\
\hline p-valor & 0,352 & 0,288 & 0,577 \\
\hline
\end{tabular}

W

Tempo na função anos

$\begin{array}{cccc}\text { Sem Dor } & 7,83(6,74) & 8,12(6,61) & 8,69(6,59) \\ \text { Com Dor } & 10,61(6,16) & 11,36(6,27) & 11(7,79) \\ \text { p-valor } & 0,049 & 0,073 & 0,504\end{array}$

W $\quad 0,248$

Teste de Mann-Whitney; W - Tamanho de efeito; $\mathrm{CH}$ - Carga Horária 


\section{DISCUSSÃO}

Este estudo se propôs a traçar o perfil funcionários do TJSE que fazem uso do computador em sua rotina de trabalho e avalia-los no que se refere aos sintomas osteomusculares e a condição ergonômica dos seus postos de trabalho. Além de observar se há correlação entre as variáveis de estudo.

As atividades laborais podem levar ao desenvolvimento de desordens em todo o sistema musculoesquelético. As regiões do corpo com os maiores índices de acometimento são pescoço, membros superiores e costas (SCHNEIDER, 2010; SILVERSTEIN, 2011).

Neste estudo, as regiões corporais com maior prevalência de sintomas osteomusculares, de acordo com o QNSO, foram Pescoço (59\%), Lombar (49\%), Torácica (46\%) e Punho/mão/dedos (41\%) (Tabela 2). Esse resultado foi observado tanto no período dos últimos 12 meses quanto nos 7 dias anteriores à entrevista. Resultado semelhante foi encontrado nos trabalhos de Oha (2014), cujo objetivo foi avaliar a prevalência de dor musculoesquelética em usuários de computador, verificando que os sintomas foram mais frequentes em pescoço, lombar, punho/mão e ombros. A manutenção da sedestação por longos períodos desencadeia fadiga muscular, déficit proprioceptivo e sobrecarga na coluna, podendo repercutir com quadros álgicos e/ou lesão nessa região (MARQUES, 2010).

Nos testes de correlação entre as variáveis, verificou-se que a média de peso corporal dos indivíduos que necessitaram de afastamento devido aos sintomas osteomusculares no pescoço foi estatisticamente superior os que não interromperam suas atividades ( $p$-valor $=$ 0,041 e $W=0,256$ ).

A idade apresentou correlação positiva com os distúrbios osteomusculares no ombro na última semana ( $p$-valor $=0,047$ e W =0,250) e nos 12 meses ( $p$-valor $=0,028$ e W =0,277) anteriores à pesquisa. Sendo a média de idade superior para os entrevistados que referiram os sintomas. Ainda na região do ombro, foi observado que os indivíduos sem dor tinham média de peso estatisticamente superior ao grupo com queixas musculoesqueléticas ( $p$ valor $=0,044$ e W $=0,273$ ). Ademais, o tempo de exercício da mesma função também se mostrou um fator relacionado com os sintomas nessa articulação. Os indivíduos que relataram distúrbios possuíam, em média, tempo maior nessa atividade laboral.

A prevalência de dor na região torácica foi estatisticamente maior no gênero feminino no período dos últimos 12 meses precedentes à pesquisa ( $p$-valor $=0,040 \mathrm{e} \mathrm{W}=0,259)$.

O ambiente de trabalho onde foram coletados os dados apresentados, neste estudo, possuiu uma padronização de estrutura entre as salas. Justificando-se assim a classificação ergonômica, conforme o Checklist, comum a quase todos os postos de trabalho, uma vez que os escores sofreram poucas alterações entre si. As maiores variações na categorização ergonômica foram encontradas nos itens referentes ao monitor utilizado. Isso se justifica por estes itens estarem diretamente relacionados com os trabalhadores que os utiliza, dependendo principalmente da estatura dos mesmos. É válido atentar, também, para o fato de os itens 3,5, 9, 10 e 12, referentes à avaliação de suporte de teclado, porta-documentos, notebook, layout e iluminação, respectivamente, não foram utilizados por conta da indisponibilidade dos materiais técnicos para realizar as medições necessárias ou por não se aplicarem ao ambiente avaliado. Entretanto, esse fato não interfere na avaliação ergonômica, visto que não há interdependência dos itens do Cheklist.

As menores pontuações registradas no Checklist foram na avaliação da cadeira e do monitor. No primeiro item, os principais problemas encontrados foram referentes ao encosto da cadeira, quanto à regulagem e firmeza no apoio da região lombar. No outro item as inadequações apresentadas foram a altura na qual se encontrava o monitor e nem sempre havia mecanismo de ajuste estava disponível ou se fazia suficiente.

Os dispositivos de regulagem presentes no mobiliário parecem não ser garantia de que os usuários o utilizem, ou o façam de maneira adequada. Além disso, durante a aplicação dos 
questionários, foi relatado que os funcionários já haviam recebido orientações de um fisioterapeuta para correção da postura sentada e os devidos ajustes nos postos de trabalho. Dessa forma, sugere-se que as visitas de um profissional de fisioterapia em menores espaços de tempo. Eerd et al (2016) sugeriu em revisão sistemática que a adoção de um programa de atividade física para os funcionário, incluindo exercícios resistidos e alongamentos, somado à utilização de mouse e apoio para antebraços podem prevenir o surgimento de problemas osteomusculares.

David (2005) realizou uma revisão sobre os diferentes meios de avaliação de risco ergonômico, como entrevistas, questionários, observação e instrumentos de medição de agentes físicos, relacionados com os distúrbios do sistema musculoesquelético. Nesse trabalho, foi verificado que o método avaliativo dependerá do propósito do estudo e que a observação do ambiente de trabalho é uma ferramenta satisfatória quando o tempo e recursos são reduzidos nas pesquisas.

É necessário atentar para as limitações observadas na presente pesquisa. O tamanho da amostra pode ser um fator de interferência dos resultados, o que exige cautela às inferências feitas sobre os achados.

Apesar de ser um instrumento padrão-ouro nas pesquisas acerca dos sintomas osteomusculares, o QNSO mostra-se uma ferramenta pouco sensível na avaliação da dor. Uma abordagem que investigue desfechos como intensidade, duração, fatores atenuantes e provocadores da dor podem tornar os estudos em saúde do trabalhador metodologicamente mais satisfatórios. Madaleine (2014) realizou uma ampla abordagem na avaliação de trabalhadores usuários de computador, verificando intensidade e duração da dor musculoesquelética por meio de autorrelatos. Seus resultados mostraram que a intensidade, duração e o maior número de pontos de dor/desconforto prevaleceram no gênero feminino.

Quanto à prática atividade física, foi percebido que somente perguntar aos voluntários se a realizam é um dado insuficiente para correlacionar o sedentarismo aos sintomas no sistema musculoesquelético. Para os próximos estudos, é sugerido registar o tipo, nível e frequência da atividade, visto que exercícios de grande intensidade podem causar lesões, gerando viés no resultado das pesquisas.

Analisando o resultado do Checklist do mobiliário, o escore gerado na avaliação da cadeira de trabalho a classificou como Condição Ergonômica Excelente. No entanto, é preciso fazer ressalvas quanto a esse resultado. Alguns quesitos deste item servem para verificar se há ou não ferramentas de regulagem nas cadeiras (ex.: altura do acento, braços e inclinação do encosto reguláveis), porém, em caso afirmativo, isto não assegura que os dispositivos sejam utilizados pelos funcionários. Fato que reforça, mais uma vez, a importância do trabalho de orientação de fisioterapeutas para otimizar o uso do mobiliário.

\section{CONCLUSÃO}

Diante do conteúdo apresentado, sugere-se que o mobiliário dos postos de trabalho, o sobrepeso dos trabalhadores e o tempo em que o funcionário exerce a mesma função, estejam relacionados com os distúrbios osteomusculares e que os achados desta pesquisa, assim como, suas limitações, sirvam de instrumentos norteadores para futuros estudos, aditando valor científico à área da saúde do trabalhador e à fisioterapia baseada em evidências.

\section{REFERÊNCIAS}

ASSOCIAÇÃO BRASILEIRA DE ERGONOMIA. O que é ergonomia? Disponível em $<$ http://www.abergo.org.br/internas.php?pg=o_que_e_ergonomia> Acesso em: 03 de janeiro de 2015. 
BEZERRA, M. L. S.; NEVES, E. B.. Perfil da produção científica em saúde do trabalhador. São Paulo, Saúde \& Sociedade, v. 19, n. 2, Jun.2010.

CONSELHO NACIONAL DE SAÚDE. Comissão Intersetorial de Saúde do Trabalhador CIST. Brasília, DF.

HUMAN FACTORS AND ERGONOMICS SOCIETY. Disponível em : https://www.hfes.org/web/AboutHFES/about.html Acesso em: 03 de janeiro de 2015.

INTERNATIONAL ERGONOMICS ASSOCIATION. Definition and Domains of ergonomics. 2015. Disponível em: http://www.iea.cc/whats/index.html. Acesso em: 03 de janeiro de 2015.

KUORINKA, I.; JONSSON, B.; KILBOM, A.; VINTERBERG, H.; BIERING-SORENSEN, F.; ANDERSSON, G. et al. Standardised Nordic questionnaires for the analysis of musculoskeletal symptoms. Applied Ergonomics, v. 18, p. 233-237, sept. 1987.

LEMOS, L. C.; MARQUEZE, E. C.; MORENO, C. R. C.. Prevalência de dores musculoesqueléticas em motoristas de caminhão e fatores associados. São Paulo, Revista Brasileira de Saúde Ocupacional, v. 39, n. 129, jun, 2014.

loskeletal symptoms. Applied Ergonomics, v. 18, p. 233-237, sept. 1987.

MARTARELLO, N. A; BENATTI, M. C. C.. Qualidade de vida e sintomas osteomusculares em trabalhadores de higiene e limpeza hospitalar. São Paulo, Revista Escola de Enfermagem da USP, v. 43, n. 2, Jun. 2009.

MÁSCULO, F. S.; VIDAL, N. C.(Org.). Ergonomia: trabalho adequado e eficiente. Rio de Janeiro: Elsevier, 2011. (Abepro). p.113.

MENDES, R.; DIAS, E. C. Da medicina do trabalho à saúde do trabalhador. São Paulo, Revista de Saúde Pública, v. 25, n. 5, Out. 1991.

PINHEIRO, F. A.; TROCCOLI, B. T.; CARVALHO, C. V. Validação do Questionário Nórdico de Sintomas Osteomusculares como medida de morbidade. São Paulo Revista de Saúde Pública v. 36, n. 3, jun. 2002 .

ROBOREDO, M. M.; POLISSENI, M. L. C. Condição ergonômica dos postos de trabalho e dor percebida de trabalhadores em escritórios da Universidade Federal de Juiz de Fora. Fisioterapia Brasil. v. 7, n.6, p. 418-422, nov./dez. 2006.

ROSA, L. et al. Avaliação da utilização do mobiliário em postos administrativos. Fisioterapia Brasil. v. 10, n. 4, p. 235-240, jul./ago. 2009.

SCHNEIDER, E., Irastorza X OSH in figures: work-related musculoskeletal disorders in the EU-facts and figures. Luxembourg: European Agency for Safety and Health at Work (EUOSHA), 2010.

SILVERSTEIN, B., EVANOFF, B. Musculoskeletal disorders. In: LEVY BS, WEGMAN DH, BARON SL, et al., edsOccupational and environmental health: recognizing and preventing disease and injury. New York, NY: Oxford University. Press: 335-65. 2011

VITTA, A. et al . Prevalência e fatores associados à dor musculoesquelética em profissionais de atividades sedentárias. Curitiba, Fisioterapia em Movimento, v. 25, n. 2, jun. 2012. 